\title{
The Halo by Halo Missing Baryon Problem
}

\author{
Stacy S. McGaugh \\ Department of Astronomy, University of Maryland, College Park, MD 20742, USA \\ email: ssm@astro.umd.edu
}

\begin{abstract}
The global missing baryon problem - that the sum of observed baryons falls short of the number expected form BBN - is well known. In addition to this, there is also a local missing baryon problem that applies to individual dark matter halos. This halo by halo missing baryon problem is such that the observed mass fraction of baryons in individual galaxies falls short of the cosmic baryon fraction. This deficit is a strong function of circular velocity. I give an empirical estimate of this function, and note the presence of a critical scale of $\sim 900 \mathrm{~km} \mathrm{~s}^{-1}$ therein. I also briefly review $\Omega_{b}$ from BBN, highlighting the persistent tension between lithium and the CMB, and discuss some pros and cons of individual galaxies and clusters of galaxies as potential reservoirs of dark baryons.
\end{abstract}

Keywords. galaxies: formation, galaxies: fundamental parameters, galaxies: clusters, dark matter, cosmic microwave background, cosmological parameters

\section{Introduction}

Modern cosmology suffers a hierarchy of missing mass problems. Most famously, there appears to be more gravitating mass than can be accounted for with baryons while maintaining consistency with Big Bang Nucleosynthesis (BBN):

$$
\Omega_{b}<\Omega_{m} .
$$

This, together with the need to grow large scale structure, leads to the inference of non-baryonic cold dark matter (CDM).

In addition to the dynamical missing mass problem, there is also the missing baryon problem (e.g., Persic \& Salucci 1992, Fukugita et al. 1998). Our inventory of known baryons in the local universe, summing over all observed stars, gas, etc., comes up short of the total anticipated from BBN:

$$
\Omega_{*}+\Omega_{g}+\ldots<\Omega_{b} .
$$

For example, Bell et al. (2003) estimate that the sum of stars and cold gas is only $\sim 3 \%$ of $\Omega_{b}$. While there now seems to be a good chance that many of the missing baryons are in the form of highly ionized gas in the warm-hot intergalactic medium (the WHIM - see Mathur, these proceedings), we are still far from being able to give a confident accounting of where all the baryons reside. Indeed, there could be multiple distinct reservoirs in addition to the WHIM, each comparable to the mass in stars, within the current uncertainties.

Here I highlight the halo by halo missing baryon problem. In addition to the global missing baryon problem, there seems to be a mismatch between the mass of baryons in individual galaxies and the mass of their host halos. For the amount of dark matter we infer dynamically, the mass in detected baryons falls short of the cosmic baryon fraction:

$$
m_{d}<f_{b} \text {. }
$$




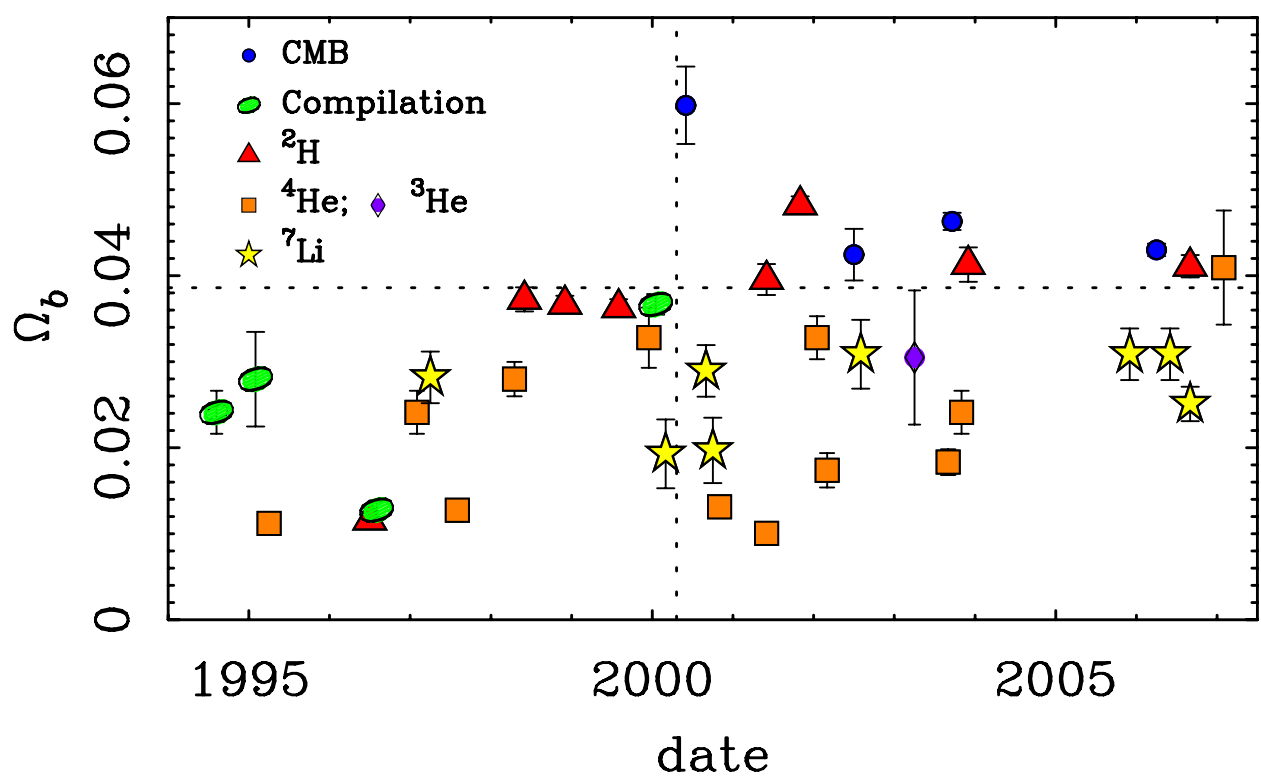

Figure 1. The baryon density from various measurements over the past decade, as tabulated by McGaugh (2004), with a few recent updates (Charbonnel \& Primas 2005, Asplund et al. 2006, Bonifacio et al. 2007, O'Meara et al. 2006, Peimbert et al. 2007, Spergel et al. 2007). Constraints from each independent method are noted by different symbols. BBN was well established long before the start date of this graph; previous work is represented by early compilations. This plot assumes $H_{0}=72 \mathrm{~km} \mathrm{~s}^{-1} \mathrm{Mpc}^{-1}$; the first point is the famous $\Omega_{b} h^{2}=0.0125$ value of Walker et al. (1991). Note that no measurement of any isotope ever suggested a value $\Omega_{b} h^{2}>0.02$ (horizontal dotted line) prior to the appearance of relevant CMB data (vertical dotted line). With the exception of the case of no CDM (McGaugh 1999, 2004), no CMB fit has ever suggested $\Omega_{b} h^{2}<0.02$. Measured values of some isotopes seem to have drifted upwards towards the CMB values since 2000 , a worrisome trend given the historical tendency for cosmological measurements to track one another.

Here

$$
m_{d}=\frac{M_{b}}{M_{v i r}}
$$

is the mass fraction of detected baryons in a given object, and the cosmic baryon fraction is $f_{b}=0.17$ (Spergel et al. 2007). Unfortunately, the virial mass of any given galaxy is a rather notional quantity, and there are many possible mappings between the observed baryonic content and the inferred dark mass. Nevertheless, there is a pronounced regularity to the data that does not tumble naturally out of our $\Lambda \mathrm{CDM}$ theory.

\section{Big Bang Nucleosynthesis}

Before proceeding with a discussion of the missing baryon problem in individual dark matter halos, it is worth reviewing $\Omega_{b}$. It is commonly stated that we have entered the era of precision cosmology. If this is true, then there is a tension between $\Omega_{b}=0.03$ and 0.04 that should concern us.

One of the great successes of cosmology is BBN. The abundance of the light elements depends on a single parameter, the baryon-to-photon ratio, that maps directly to the baryon density. Independent measurements of ${ }^{2} \mathrm{H},{ }^{4} \mathrm{He}$, and ${ }^{7} \mathrm{Li}$ all indicate nearly the same value for $\Omega_{b}$. In addition, modern observations of the cosmic microwave background (CMB) provide another similar constraint on the baryon density. 
Table 1. $\Omega_{b}$ by method*.

\begin{tabular}{|c|c|c|c|c|c|c|c|}
\hline & $\mathrm{W} 91^{\dagger}$ & ${ }^{2} \mathrm{H}$ & ${ }^{4} \mathrm{He}$ & ${ }^{7} \mathrm{Li}$ & $\mathrm{CMB}$ & $\mathrm{W} 3^{\ddagger}$ & ${\mathrm{M} 04^{\star}}^{\star}$ \\
\hline$\Omega_{b}$ & 0.024 & 0.038 & 0.021 & 0.028 & 0.046 & 0.0430 & 0.033 \\
\hline$\sigma$ & 0.005 & 0.005 & 0.008 & 0.005 & 0.007 & 0.0014 & 0.006 \\
\hline
\end{tabular}

The consistency of the independent BBN constraints is impressive, and I have little doubt that the basic picture is correct. However, the agreement is not perfect. There seems to be a modest dichotomy between each of the independent methods (Fig. 1), with each preferring a slightly different value of $\Omega_{b}$. Table 1 shows a comparison of baryon densities by method. Values are the median for each case from the compilation of McGaugh 2004 assuming $H_{0}=72 \mathrm{~km} \mathrm{~s}^{-1} \mathrm{Mpc}^{-1}$. The uncertainty is taken from the variance of different published measurements. This tends to overstate the error claimed by individual determinations. For this uncertainty, the light elements are broadly consistent. If we look only at the latest WMAP result (Spergel et al. 2007), its tiny uncertainty makes it difficult to reconcile with the helium and lithium. I briefly review each method below.

Helium: Measured in very low metallicity extragalactic HII regions (e.g., Kuzio de Naray et al. 2004), helium gives the lowest value of $\Omega_{b}$. Though consistently in the vicinity of $Y_{p}=0.24$, the weak dependence of $Y_{p}$ on $\Omega_{b}$ makes it difficult to obtain a precise estimate from helium. Moreover, independent determinations vary by more than they should for the stated errors. This is likely due to the many subtle systematic effects that afflict helium abundance determinations at the $\sim 1 \%$ level (Olive \& Skillman 2004). Thus, while the broad consistency of helium with other determinations is comforting, it seems unlikely that it can provide a strong constraint on the precise value of $\Omega_{b}$. We should not take this as a free pass to simply ignore helium; all determinations of $Y_{p}$ up to the date of my review gave $\Omega_{b}<0.035$, and I found it increasingly difficult to reconcile any of the helium data with $\Omega_{b}>0.04$ (but see Peimbert et al. 2007).

Lithium: Measured in low metallicity stars (e.g., Ryan et al. 2000), lithium gives the next lowest value for $\Omega_{b}$. Unlike helium, the ${ }^{7} \mathrm{Li}$ abundance seems to be both well measured and consistent between independent determinations. The Spite plateau is sharply defined by dozens of low metallicity stars. At $\Omega_{b}=0.028$, it is marginally consistent with deuterium but not consistent with the CMB. The general presumption seems therefore to be that lithium needs to be 'fixed' (e.g., Korn et al. 2006, Piau et al. 2006), but there is nothing broken about it if the $\mathrm{CMB}$ value is overestimated or its uncertainty underestimated.

Deuterium: Of the light elements, deuterium is the most sensitive to $\Omega_{b}$. Its measurement in Lyman absorption clouds in the spectra of high redshift quasars provides one of the most accurate constraints on BBN. Indeed, it came in at surprisingly low $\mathrm{D} / \mathrm{H}$, giving high $\Omega_{b} h^{2}=0.019 \pm 0.0012$ (Tytler et al. 1999) relative to long-standing results (e.g., $\Omega_{b} h^{2}=0.0125 \pm 0.0025$ : Walker et al. 1991). Unfortunately, there exist only 6 accurate, published $\mathrm{D} / \mathrm{H}$ values (fewer systems than there are points in Fig. 1), one of which is discrepantly high.

CMB: The microwave background provides another constraint on $\Omega_{b}$ that is independent of abundance measurements. It gives a value for $\Omega_{b}$ that is close to but persistently higher than all of the light elements. The power spectrum of the CMB is very sensitive to both the absolute density of baryons $\left(\Omega_{b}\right)$ and the global baryon fraction $\left(f_{b}\right)$. Fig. 2 


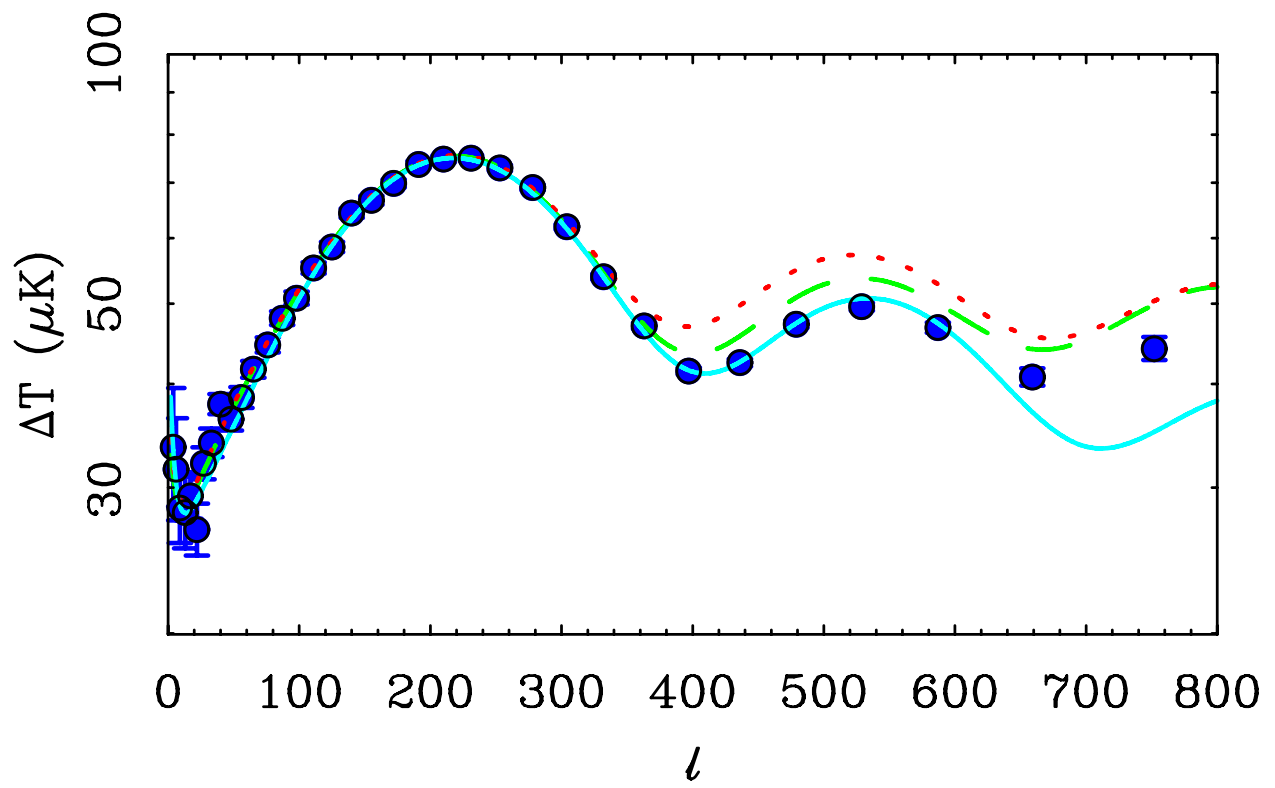

Figure 2. The effects of the baryon density and baryon fraction on the CMB. The points are the third year WMAP data (Spergel et al. 2007). The various lines are models with $\Omega_{b}$ and $f_{b}$ set to fixed values (see text). The dotted (topmost) line is set to the lithium value $\left(\Omega_{b}=0.028\right)$ with $f_{b}=0.12$ (case 1$)$. The dashed (middle) line is set to the deuterium value $\left(\Omega_{b}=0.038\right)$ with $f_{b}=0.17$ (case 2). Case 3 is the solid (bottom) line with lithium $\Omega_{b}$ and no CDM (McGaugh 1999, 2004). The CMB is exquisitely sensitive to both the absolute baryon density and the baryon fraction, but also suffers degeneracies between these and other parameters (especially the tilt).

illustrates this sensitivity with three cases: (1) a plausibly low baryon density, low baryon fraction universe; (2) a high baryon density, high baryon fraction universe, and (3) a low density, no-CDM universe.

Case (1) is motivated by the lithium baryon density (Table 1). For this value of $\Omega_{b}$, $f_{b}=0.12$ as observed in clusters (McCarthy et al. 2007; see also Holder, these proceedings) if $\Omega_{m}=0.23$, consistent with peculiar velocity measurements $\left(\Omega_{m}=0.22 \pm 0.02\right.$ : Moyahee \& Tully 2005). These three independent lines of evidence provide a self consistent and reasonable set of cosmological constraints. However, they badly overestimate the amplitude of the second acoustic peak. This problem is generic to all concordance $\Lambda \mathrm{CDM}$ models prior to the first measurement of the second peak in 2000 .

Case (2) utilizes the deuterium abundance, which gives the highest $\Omega_{b}$ of all the BBN elements and the one most consistent with fits to the CMB. A baryon fraction $f_{b}=0.17$ is adopted, giving a plausible $\Omega_{m}=0.26$. Though rigged to be as consistent as possible with CMB fits without simply adopting them, this case still over-predicts the amplitude of the second peak, albeit by a lesser amount than case (1).

Case (3) uses the lithium $\Omega_{b}$ with zero CDM. Consideration of this model is motivated by MOND, but uses entirely conventional physics. It provides quite a good fit to the second peak. Indeed, this was the only successful a proiri prediction of its amplitude (McGaugh 1999). Here it merely illustrates the sensitivity to $f_{b}$ as well as $\Omega_{b}$.

Like all models devoid of CDM, case (3) under-predicts the amplitude of the third peak, just as un-tilted $\Lambda$ CDM models over-predict it (e.g., cases 1 and 2). This 'low' third peak leads to the inference of a tilt $(n \approx 0.95)$, which in turn impacts the inferred baryon density, somewhat reducing it relative to previous CMB determinations. In the 
context of MOND, the 'high' third peak implies a driving term analogous to that provided by CDM. This occurs naturally in TeVeS-like relativistic MOND theories (Skordis et al. 2006), but it is unclear how this might affect $\Omega_{b}$.

\subsection{BBN summary}

BBN is undoubtedly one of the shining successes of modern cosmology. There is broad agreement between multiple independent lines of evidence to an accuracy rarely witnessed in cosmology. I have little doubt that the basic picture is correct.

In detail, there are significant differences between independent methods. It is common to point to the great consistency between $\Omega_{b}$ from BBN and the CMB as support for the $\Lambda$ CDM picture. However, this is rather sloppy, being only really true for deuterium. Lithium and helium prefer noticeably lower values for the baryon density.

The light elements themselves provide a self-consistent picture if we adopt the uncertainties listed in Table 1 . It is the tiny uncertainty in the CMB determination that is problematic. The CMB is indeed that sensitive (Fig. 2), but does suffer from parameter degeneracies, in particular the tilt and the baryon fraction. The tension between the light elements and the CMB disappears if there is no CDM.

It appears that the true baryon density is not yet as precisely known as might be hoped. Nevertheless, the missing baryon problem remains an issue at low redshift. Whether the true baryon density is $3 \%$ of critical or $4 \%$ depends on whether one gives more weight to light element measurements or CMB fits. Either way, $\Omega_{b}$ exceeds the well-determined inventory of local baryons (Fukugita et al. 1998).

\section{The baryon content of individual dark matter halos}

As well as trying to account for all the baryons in the universe, and the integrated amount in galaxies, clusters, and the IGM (see Frenk; Moore, these proceedings), it would be nice to have an accounting in individual systems. Each dark matter halo can, to a first approximation, be thought of as a microcosm of the whole. As such, one would naively expect each halo to have the same baryon fraction as the whole universe. On the scale of clusters of galaxies, this is approximately true. For individual galaxies, observations depart from this ideal in a way which we have yet to understand.

\subsection{The Milky Way}

Our own galaxy provides an illustration of the general problem. We have a rather complete inventory of local baryons that appears to be fairly consistent between independent estimates. Yet when we compare the baryonic mass to the total dynamical mass, it falls well short of the universal baryon fraction.

For illustration, I adopt estimates of the baryonic mass from the recent work of Flynn et al. $(2006): \mathrm{M}_{*}^{M W} \approx 5 \times 10^{10} \mathrm{M}_{\odot}$ and $\mathrm{M}_{g}^{M W} \approx 10^{10} \mathrm{M}_{\odot}$. Most of the stellar mass is in the disk, though the spit between disk and bulge depends somewhat on details like the disk scale length. The gas mass is split between molecular and atomic components. Other baryonic components appear to be negligible. The total baryonic mass of the Milky Way is thus $\mathrm{M}_{b}^{M W} \approx 6 \times 10^{10} \mathrm{M}_{\odot}$.

The baryonic masses of satellite galaxies within the Milky Way halo add little to this sum. Their total is probably less than the uncertainty in the Milky Way mass, and certainly less than $10^{10} \mathrm{M}_{\odot}$. It is thus hard to imagine that the baryonic mass associated with the Milky Way halo is any larger than $10^{11} \mathrm{M}_{\odot}$.

The total mass of the Milky Way has been studied many times. The answer persistently comes back in the vicinity of $\mathrm{M}_{t o t}^{M W} \approx 2 \times 10^{12} \mathrm{M}_{\odot}$. There are many uncertainties in this 


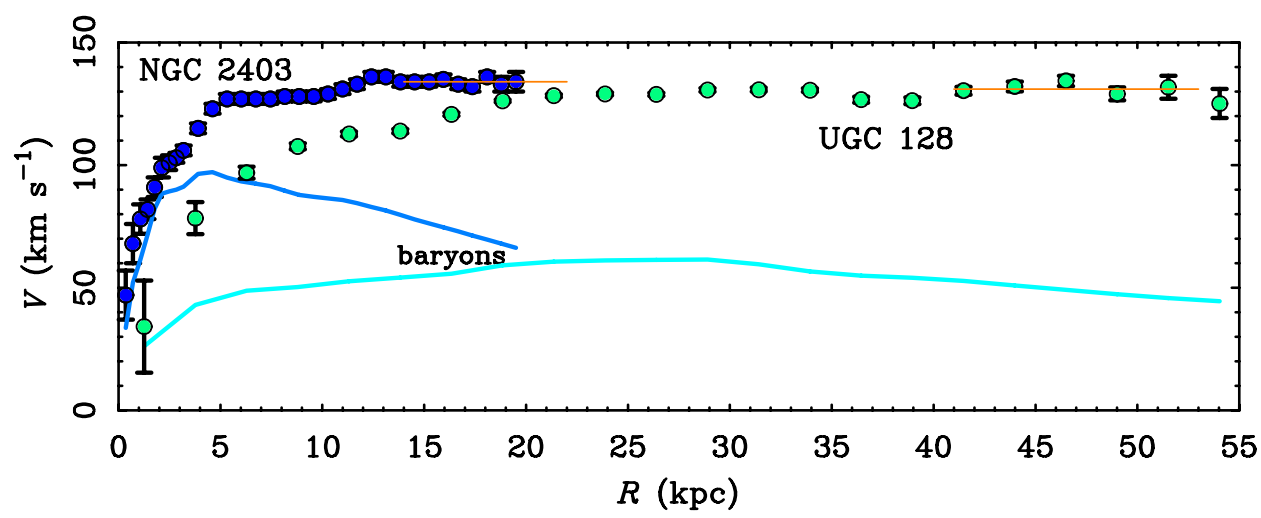

Figure 3. Rotation curves for two galaxies illustrating the circular velocity measured from the outer regions of extended $21 \mathrm{~cm}$ data (horizontal lines). The distribution of the baryonic component is also illustrated. For the purposes of this review, we are only concerned with the total mass in baryons and the characteristic circular velocity of the system.

estimate, the dominant one being whether the most distant tracer, Leo I, is considered to be bound to the Milky Way or not. Sakamoto et al. $(2003)$ give $2.5 \times 10^{12} \mathrm{M}_{\odot}$ with Leo I and $1.8 \times 10^{12} \mathrm{M}_{\odot}$ without it. A practical lower limit is $\sim 10^{12} \mathrm{M}_{\odot}$; half this is already encompassed within the radius of the LMC.

Taking these numbers at face value,

$$
m_{d}^{M W}=\frac{6 \times 10^{10} \mathrm{M}_{\odot}}{2 \times 10^{12} \mathrm{M}_{\odot}}=0.03 .
$$

This falls well short of the universal baryon fraction, $f_{b}=0.17$. Pushing the numbers to their extremes, we can place the limit $m_{d}^{M W}<0.10$. This is at least within hailing distance of $f_{b}=0.12$ from clusters, but still well short of the CMB value.

This raises the question: where are all the baryons that should be associated with the Milky Way halo? Perhaps they have been expelled, though the Milky Way is generally thought to be too massive to have suffered much of this. Another possibility is that they lurk in the halo in some as yet undetected form, like a local WHIM. While the WHIM is a great candidate for many of the globally missing baryons (see Mathur, these proceedings), it appears to fall well short of what is needed to explain this deficit in the Milky Way (Pildis \& McGaugh 1996), providing perhaps $\sim 10^{10} \mathrm{M}_{\odot}$ (Collins et al. 2005).

The Milky Way is not unique in having $m_{d}<f_{b}$. Similar discrepancies are observed in many systems (McGaugh \& de Blok 1998). Indeed, $m_{d}$ appears to deviate systematically from $f_{b}$ by an amount which becomes increasingly severe in systems of decreasing circular velocity.

\subsection{The Baryonic Tully-Fisher Relation}

A basic tenet of CDM theory is a relation between halo mass and circular velocity at the virial radius (Steinmetz \& Navarro 1999) of the form

$$
M_{v i r} \propto V_{v i r}^{3}
$$

with the normalization depending on the cosmology (Mo \& Mao 2004). We can measure neither of these quantities. What we can measure is the baryonic mass of a given galaxy and a characteristic circular velocity (Fig. 3). 


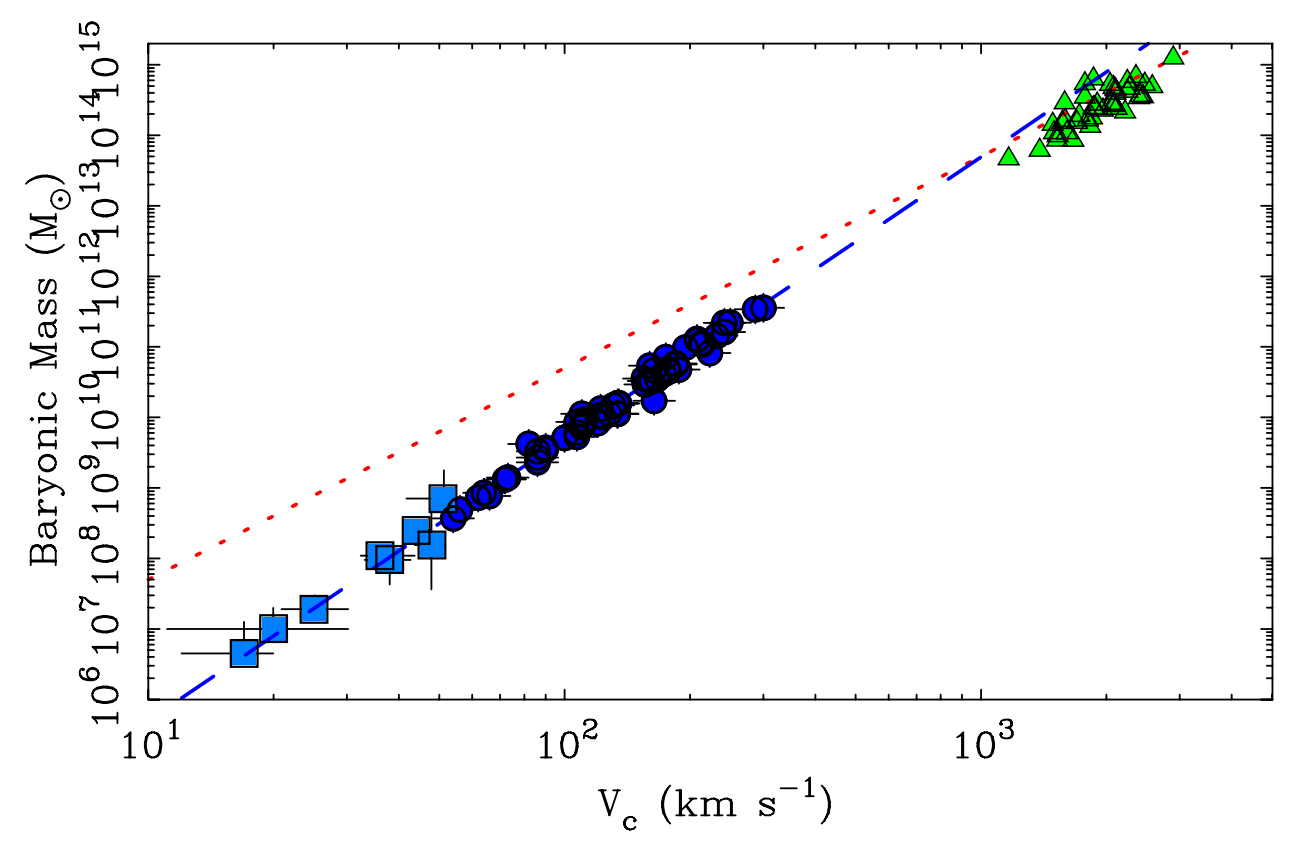

Figure 4. The baryonic mass-circular velocity relation, spanning the range from the tiniest dwarfs (squares) through spiral galaxies (circles; McGaugh 2005) to clusters of galaxies (triangles; Reiprich 2001). The dashed line is the fit to the galaxy (circles only) data; the dotted line is what we expect in $\Lambda \mathrm{CDM}$ if all the baryons in each halo are identified.

Summing up the mass in stars and gas for a large sample of spiral galaxies, we obtain the Baryonic Tully-Fisher relation:

$$
M_{b}=50 V_{c}^{4}
$$

with $M_{b}$ in solar masses and $V_{c}$ in $\mathrm{kms}^{-1}$ (Fig. 4). For an extensive discussion of the effects of various choices for the stellar mass-to-light ratio on the normalization, slope, and scatter in this relation, see McGaugh (2005). Equation 3.3 does well when extrapolated to predict the locations of systems with $V_{c}<50 \mathrm{~km} \mathrm{~s}^{-1}$, including both rotating gas rich dwarf Irr systems (the squares in Fig. 4) and pressure supported, star dominated dwarf spheroidals (Mateo \& Walker; Geha, private communications).

Extrapolation of the Baryonic Tully-Fisher relation to the cluster scale does less well, over-predicting the median cluster mass by a factor of $\sim 2.5$. Perhaps these are simply different systems, and such a vast extrapolation is unwarranted. On the other hand, coming this close with an extrapolation over several decades in mass is fairly remarkable.

That clusters fall a little below the extrapolation of the Baryonic Tully-Fisher relation may suggest that our census of baryons is not complete in these systems. If so, clusters might be a good place to look for some of the missing baryons. Clusters are not a large contributor to the total integrated baryonic mass of the universe, so even if more baryons are lurking there, they are not likely to solve the missing baryon problem. Placing them on the extrapolation of equation 3.3 would require (very roughly) $5 \%$ of $\Omega_{b}$. While not a large fraction of the total, this is comparable to all the stars in field galaxies. Most of the missing baryons could be in the WHIM and still leave room for a number of other distinct baryonic reservoirs like this.

The CDM mass-velocity relation comes much closer to the cluster data than does the empirical Baryonic Tully-Fisher relation. The difference between it and the median cluster 


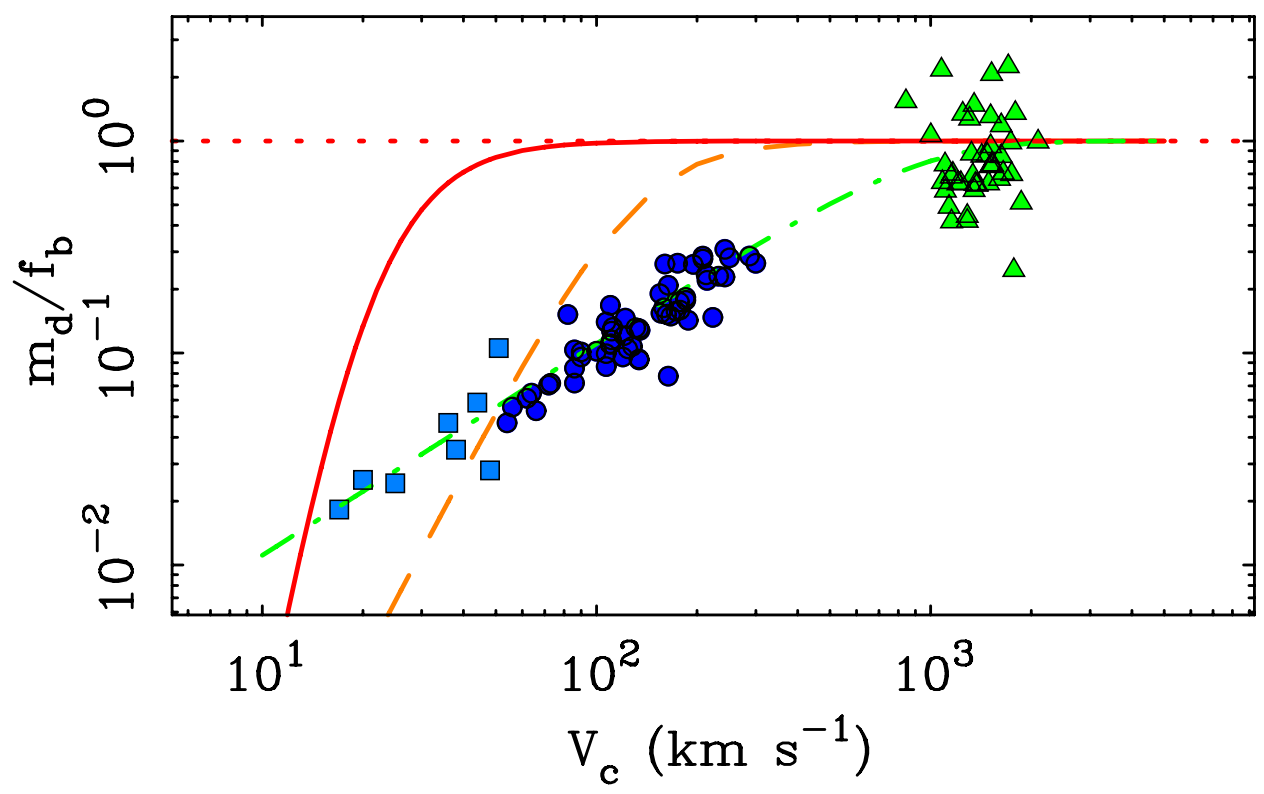

Figure 5. The baryon content as a function of circular velocity. Points are galaxy and cluster data, as per Fig. 4. The solid line is the reionization model from Crain et al. (2007) (see also Mayer \& Moore 2004); the dashed line is the preheating model of Mo \& Mao (2004); the dash-dot line is a fit to the data (see text).

value is the difference between $f_{b}=0.17$ and 0.12 . This near agreement deteriorates for smaller systems. The slope of the halo mass-virial velocity relation is shallower than the observed baryonic mass-circular velocity relation. Even bearing in mind the caveat that the halo virial and observed circular velocities may not be simply related, it is difficult to avoid the conclusion that the observed number of baryons is systematically less than the expected number as one goes down the mass function.

If we take the halo mass-virial velocity relation as sacrosanct, then it is impossible to avoid the conclusion that $m_{d}$ varies with $V_{c}$ (Fig. 5). Empirically, this variation is well fit by

$$
m_{d}=f_{b} \tanh \left(\frac{V_{c}}{V_{\dagger}}\right) .
$$

Here $V_{\dagger}$ is a scale where $m_{d} \rightarrow f_{b}$; the dash-dot line shown in Fig. 5 uses equation 3.4 with $V_{\dagger}=900 \mathrm{~km} \mathrm{~s}^{-1}$. This is the scale where the dashed and dotted lines cross in Fig. 4 which is where the data transition from looking like $\Lambda \mathrm{CDM}$ on larger scales to looking like MOND on smaller scales. Note that something odd is happening on intermediate scales, as groups in the Local Volume imply $\Omega_{m} \approx 0.04$ (Karachentsev 2005). (Yes, that is $\Omega_{m}$, not $\Omega_{b}$.)

In order to derive equation 3.4 or plot Fig. 5 , it is necessary to make some assumption in order to relate total halo mass to observed baryonic mass. For simplicity, I assume $V_{v i r}=V_{c}$ here. While I do not expect this to hold in detail, plausible adjustments (due, for example, to adiabatic compression) will only tweak the details and not alter the basic result. That equation 3.4 returns a value consistent with that for the Milky Way (equation 3.1) suggests that the simple assumption is not terrible.

The variation of $m_{d}$ with $V_{c}$ implies that there should be missing baryons associated with each and every galaxy halo (see also Zackrisson, these proceedings). Equation 3.4 
is merely a fit to the data. What should we expect? Recent work gives some idea: Mayer \& Moore (2004) and Crain et al. (2007) consider the effects of reionization while Mo \& Mao (2004) consider the possibility of preheating in analogy with clusters. Unfortunately, neither of these types of models matches the observed trend (Fig. 5). In particular, there is no evidence in the data for the sudden transition expected around $\sim 20 \mathrm{~km} \mathrm{~s}^{-1}$ from reionization.

In the context of $\Lambda \mathrm{CDM}$, this merely means that there is more physics than reionization mediating between the primordial mass-velocity relation and the observed one. Feedback is frequently invoked in this context, but this word has come to mean many different things. It remains to be demonstrated whether some flavor of feedback can be a solution to all these problems.

There are, in principle, many potential sources of scatter that should manifest themselves in the Tully-Fisher relation (McGaugh \& de Blok 1998), yet the observed relation has remarkably little scatter. It is hard to imagine how $m_{d}$ can vary so much between bright and faint galaxies with virtually zero scatter along the way. Somehow, a $V_{c} \approx 50 \mathrm{~km} \mathrm{~s}^{-1}$ galaxy must 'know' it should have $m_{d} \approx 0.01$ and never 0.04 , while for a galaxy with $V_{c} \approx 220 \mathrm{~km} \mathrm{~s}^{-1}$ the situation is reversed. The small observed scatter only happens naturally if $m_{d}=f_{b}$ (McGaugh et al. 2000). This implies that essentially all the baryons are accounted for on galaxy scales, making individual galaxies an unlikely place to look for the missing baryons. However, this contradicts a basic tenet of CDM, as the intrinsic mass-velocity relation differs from equation 3.2 if $m_{d}=f_{b}$.

\section{Conclusions}

I provide two sets of interpretations, one in the context of $\Lambda \mathrm{CDM}$, the other from an empirical perspective.

\subsection{Conclusions in the context of $\Lambda C D M$}

- There is no signature in the data due to reionization in the vicinity of $\sim 20 \mathrm{~km} \mathrm{~s}^{-1}$. Low mass galaxies smoothly follow the extrapolation of the Baryonic Tully-Fisher relation fit to galaxies with $V_{c}>50 \mathrm{~km} \mathrm{~s}^{-1}$.

- The baryon content of halos scales with circular velocity as summarized by equation 3.4 .

- Models invoking reionization or pre-heating fail to match the observed trend in $m_{d}$.

- Physics beyond reionization is required to explain the observations.

In order to reconcile the data with $\Lambda \mathrm{CDM}$, some physical effect must make the observed baryon fraction $m_{d}$ the observed function of circular velocity. Whatever mechanism is invoked must operate with remarkably small scatter, posing a fine-tuning problem. The physics of galaxy formation - the bridge connecting the primordial halos found in numerical simulations with real observed galaxies - remains as sound and secure as the Bridge of Death over the Gorge of Eternal Peril in Monty Python's Holy Grail.

\subsection{Empirical conclusions}

- The observed mass-velocity relation is steeper than predicted by CDM.

- The small scatter in the Baryonic Tully-Fisher relation implies that substantially all the baryonic mass has been accounted for in individual galaxies.

- The two preceding items contradict a basic tenet of CDM.

- Extrapolation of the Baryonic Tully-Fisher relation to cluster scales suggests that the inventory of baryons in clusters may be incomplete.

The last point implies that perhaps some of the missing baryons are hiding in clusters in some yet to be identified form. These need not be a large fraction of the missing baryons, 
maybe $5 \%$. There is precedence for such a situation. Nowadays it is widely accepted that the hot X-ray emitting intra-cluster gas is the dominant baryonic component of rich clusters. A couple of decades ago, stars were widely thought to be the dominant baryonic component. Though I am at a loss to suggest a plausible state in which baryons might hide (but see Bonamente et al. 2003), this history reminds us that we should not be over-confident about the non-existence of matter we haven't yet detected.

\section{Acknowledgements}

This material is based upon work supported by the National Science Foundation under Grant No. 0505956. I would like to thank the organizers, Mike and Jon in particular, for putting together this delightful conference.

\section{References}

Asplund, M., Lambert, D. L., Nissen, P. E., Primas, F., \& Smith, V. V. 2006, ApJ, 644, 229 Bonifacio, P., et al. 2007, A\& $A, 462,851$

Bell, E. F., McIntosh, D. H., Katz, N., \& Weinberg, M. D. 2003, ApJ, 585, L117

Bonamente, M., Joy, M. K., \& Lieu, R. 2003, ApJ, 585, 722

Charbonnel, C., \& Primas, F. 2005, A\&GA, 442, 961

Collins, J. A., Shull, J. M., \& Giroux, M. L. 2005, ApJ, 623, 196

Crain, R. A., Eke, V. R., Frenk, C. S., Jenkins, A., McCarthy, I. G., Navarro, J. F., \& Pearce, F. R. 2007, MNRAS, 377, 41

Flynn, C., Holmberg, J., Portinari, L., Fuchs, B., \& Jahreiß, H. 2006, MNRAS, 372, 1149

Fukugita, M., Hogan, C., \& Peebles, P. J.E. 1998, ApJ, 503, 518

Karachentsev, I. D. 2005, AJ, 129, 178

Korn, A. J., Grundahl, F., Richard, O., Barklem, P. S., Mashonkina, L., Collet, R., Piskunov, N., \& Gustafsson, B. 2006, Nature, 442, 657

Kuzio de Naray, R., McGaugh, S. S., \& de Blok, W. J. G. 2004, MNRAS, 355, 887

Mayer, L., \& Moore, B. 2004, MNRAS, 354, 477

McCarthy, I. G., Bower, R. G., \& Balogh, M. L. 2007, MNRAS, 377, 1457

McGaugh, S. S. 1999, ApJ, 523, L99

McGaugh, S. S. 2004, ApJ, 611, 26

McGaugh, S. S. 2005, ApJ, 632, 859

McGaugh, S. S., \& de Blok, W. J. G. 1998, ApJ, 499, 41

McGaugh, S. S., Schombert, J. M., Bothun, G. D., \& de Blok, W. J. G. 2000, ApJ, 533, L99

Mo, H. J., \& Mao, S. 2004, MNRAS, 353, 829

Mohayaee, R., \& Tully, R. B. 2005, ApJ, 635, L113

Olive, K. A., \& Skillman, E. D. 2004, ApJ, 617, 29

O'Meara, J. M., Burles, S., Prochaska, J. X., Prochter, G. E., Bernstein, R. A., \& Burgess, K. M. 2006, $A p J, 649$, L61

Peimbert, M., Luridiana, V., \& Peimbert, A. 2007, ApJ, in press (astro-ph/0701580)

Persic, M., \& Salucci, P. 1992, MNRAS, 258, P14

Piau, L., Beers, T. C., Balsara, D. S., Sivarani, T., Truran, J. W., \& Ferguson, J. W. 2006, ApJ, 653, 300

Pildis, R. A., \& McGaugh, S. S. 1996, ApJ, 470, L77

Reiprich, T. H. 2001, PhD thesis, Ludwig-Maximilians-Universität

Ryan, S. G., Beers, T. C., Olive, K. A., Fields, B. D., \& Norris, J. E. 2000, ApJ, 530, L57

Sakamoto, T., Chiba, M., \& Beers, T. C. 2003, A $₫ A, 397,899$

Skordis, C., Mota, D. F., Ferreira, P. G., \& Boehm, C. 2006, Phys. Rev. Lett. 96, 011301

Spergel, D. N. et al. 2007, ApJS, 170, 377

Steinmetz, M., \& Navarro, J. F. 1999, ApJ, 513, 555

Tytler, D., O'Meara, J. M., Suzuki, N., \& Lubin, D. 1999, Physics Reports, 333, 409

Walker, T. P., Steigman, G., Kang, H., Schramm, D. M., \& Olive, K. A. 1991, ApJ, 376, 51 